\title{
Activation of CXCL-8 Transcription by Hepatitis E Virus ORF-1 via AP-1
}

\author{
Zhubing Li, ${ }^{1}$ Lu Chen, ${ }^{2,3}$ and Qiang Liu ${ }^{4}$ \\ ${ }^{1}$ VIDO-InterVac, Vaccinology and Immunotherapeutics Program, University of Saskatchewan, Saskatoon, SK, Canada S7N 5E3 \\ ${ }^{2}$ VIDO-InterVac, University of Saskatchewan, Saskatoon, SK, Canada S7N 5E3 \\ ${ }^{3}$ Henan Agricultural University, 95 Wenhua Road, Zhengzhou, Henan 450002, China \\ ${ }^{4}$ VIDO-InterVac, Vaccinology and Immunotherapeutics Program, Veterinary Microbiology, University of Saskatchewan, \\ Saskatoon, SK, Canada S7N 5E3
}

Correspondence should be addressed to Qiang Liu; qiang.liu@usask.ca

Received 12 March 2015; Accepted 22 April 2015

Academic Editor: Limin Chen

Copyright (C) 2015 Zhubing Li et al. This is an open access article distributed under the Creative Commons Attribution License, which permits unrestricted use, distribution, and reproduction in any medium, provided the original work is properly cited.

Hepatitis E virus (HEV) is a small nonenveloped single-stranded positive-sense RNA virus and is one of the major causes for acute hepatitis worldwide. CXCL-8 is a small multifunctional proinflammatory chemokine. It was reported recently that HEV infection significantly upregulates CXCL- 8 gene expression. In this study, we investigated the mechanism of HEV-induced CXCL8 transcriptional activation. Using CXCL- 8 promoter reporters of different lengths ranging from -1400 to -173 , we showed that -173 promoter has the highest promoter activity in the presence of HEV genomic RNA, indicating that the -173 promoter contains sequences responsible for CXCL- 8 activation by HEV. Ectopic expression of the ORF-1 protein can upregulate the -173 CXCL-8 promoter activity. In contrast, expression of the ORF-2 protein suppresses the CXCL-8 promoter activity and expression of the ORF-3 protein has no effect on the CXCL-8 promoter activity. We further showed that AP-1 is required for CXCL-8 activation because neither HEV genomic RNA nor the ORF-1 protein can upregulate the -173 CXCL- 8 promoter in the absence of the AP-1 binding sequence. Taken together, our results showed that HEV and HEV ORF-1 protein activate the CXCL-8 promoter via AP-1. This novel function of HEV ORF-1 protein should contribute to our understanding of HEV-host interactions and HEV-associated pathogenesis.

\section{Introduction}

Hepatitis E virus (HEV), first identified in 1983, is a small nonenveloped single-stranded positive-sense RNA virus. It belongs to the genus Hepevirus in the Hepeviridae family and is classified into four genotypes [1, 2]. According to WHO, about two billion people are estimated to be infected with HEV with 14 million people showing clinical symptoms $[3,4]$. HEV mainly causes self-limiting acute hepatitis, but the mortality rate is as high as $15-25 \%$ among pregnant women. Chronic hepatitis develops exclusively in immunocompromised patients infected with genotype 3 or $4 \mathrm{HEV} \mathrm{[5-7].}$

HEV genome is approximately $7.2 \mathrm{~kb}$ and consists of three partially overlapping open reading frames (ORFs) flanked by short $5^{\prime}$ and $3^{\prime}$ untranslated regions $[8,9]$.
ORF-1 encodes a nonstructural polyprotein that can be cleaved into methyltransferase, papain-like cysteine protease, RNA helicase, and RNA-dependent RNA polymerase, which are essential for virus replication. ORF-2 encodes the viral capsid protein which contains neutralizing epitopes and ORF-3 encodes a small phosphoprotein that functions in virion morphogenesis, release, and pathogenesis [10-12].

CXCL-8, also known as interleukin-8, is a small multifunctional proinflammatory chemokine in the CXC chemokine family, which plays an important role in immune defence processes [13-15]. CXCL-8 is secreted at the inflammatory sites by different cells, such as neutrophils, macrophages, monocytes, and endothelial cells. CXCL-8 primarily targets the polymorphonuclear leukocytes which act as the first line of immune defence $[16,17]$. Furthermore, CXCL-8 can also 
induce tumorigenesis, metastasis, and angiogenesis [17, 18]. CXCL-8 gene transcription can be regulated by NF- $\kappa$ B and activator protein-1 (AP-1) [19].

Since it was recently shown that HEV infection significantly upregulates CXCL-8 gene expression [20], we investigated how HEV mediates CXCL-8 transcriptional activation. We showed that HEV genomic RNA and the ORF-1 protein upregulated CXCL-8 promoter-mediated transcription via AP-1.

\section{Material and Methods}

2.1. Plasmids. A cell culture adapted HEV genotype 3 Kernow $\mathrm{C} 1$ strain cDNA clone was received from Dr. Emerson [21]. The coding sequences of ORF-1, ORF-2, and ORF-3 were cloned into a pEF $3 x$ Flag vector. Human CXCL-8 promoterluciferase reporters of different lengths, $-1400,-500,-230$, -193 , and -173 , were received from Dr. Buendia [22]. Mutant -173 CXCL-8 promoters with mutations for the AP-1 binding sequence $\left({ }^{-126} \mathrm{TGACTCA}^{-120}\right.$ to $\left.{ }^{-126} \mathrm{TatCTCA}^{-120}, \mathrm{mAP}-1\right)$ [23] or the negative regulatory cis-element sequence (NRE) $\left({ }^{-76}\right.$ ATTTCCTCTGA ${ }^{-66}$ to ${ }^{-76}$ ATTTCCcCcGA ${ }^{-66}$, mNRE) [24] were generated by site-directed mutagenesis. The AP-1luciferase reporter was generated by inserting seven tandem repeats of the AP- 1 binding sequence into the pGL4.27 vector (Promega). All plasmids were verified by DNA sequencing.

2.2. In Vitro Transcription. HEV genomic RNA was produced by in vitro transcription from linearized HEV cDNA using the mMESSAGE mMACHINE T7 kit (Ambion).

2.3. Cell Lines and Transfection. Human hepatoma HuH-7 and human embryonic kidney HEK293T cells were cultured in Dulbecco's Modified Eagle Medium (DMEM, SigmaAldrich) with $10 \%(\mathrm{v} / \mathrm{v})$ fetal bovine serum (FBS, Life Technologies) at $37^{\circ} \mathrm{C}$ and $5 \% \mathrm{CO}_{2}$. For luciferase assays, cells in 24-well plates were cotransfected with $1 \mu \mathrm{g}$ of HEV RNA and $1 \mu \mathrm{g}$ of luciferase reporter DNA using the jetPEI reagent (Polyplus), or with $0.5 \mu \mathrm{g}$ of plasmid DNA expressing HEV ORF proteins and $0.5 \mu \mathrm{g}$ of luciferase reporter DNA using the calcium phosphate precipitation method [25]. In the immunoprecipitation experiments, cells in 6-well plates were transfected with $2 \mu \mathrm{g}$ of plasmid DNA expressing HEV ORF proteins.

2.4. Luciferase Assay. Cells were lysed in Passive Lysis Buffer (Promega) and the luciferase activity was measured using a Luciferase Assay System (Promega). Luciferase activity was normalized to the protein concentration quantified using the Bradford assay reagent (Bio-Rad).

2.5. Immunoprecipitation, Western Blotting, and Antibodies. Cell lysates in RIPA buffer were immunoprecipitated with an anti-Flag antibody (Sigma-Aldrich) using the SureBeads magnetic beads system (Bio-Rad). Precipitated proteins were subjected to SDS-PAGE and transferred to nitrocellulose membranes. The blots were blocked in 5\% skim milk in PBS for 1 hour and incubated with the anti-Flag antibody

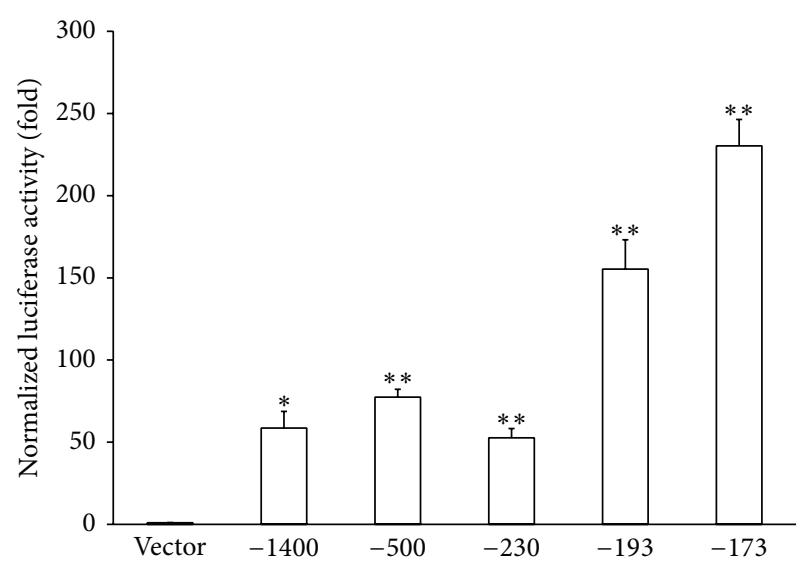

FIGURE 1: CXCL-8 promoter activities in the presence of HEV genomic RNA. HuH-7 cells were cotransfected with HEV genomic RNA and CXCL-8 promoter-luciferase reporters of different lengths or a luciferase reporter vector without the CXC-8 promoter. Luciferase assay was performed 48 hours after transfection. Luciferase activity was normalized against the protein concentration of the same samples. $*: p \leq 0.05 ; * *: p \leq 0.01$ by Student's $t$-test.

overnight at $4^{\circ} \mathrm{C}$. After washing with $\mathrm{PBS}$, the blots were incubated with an infrared dye-labeled secondary antibody (Li-Cor Biosciences) for 1 hour at room temperature and then washed again. The blot was scanned using Odyssey Infrared Imaging System (Li-Cor Biosciences).

2.6. Statistical Analysis. All experiments were performed three times in triplicate and differences between samples were assessed by Student's $t$-test. A $p$ value less than 0.05 was considered statistically significant.

\section{Results}

3.1. Determination of CXCL-8 Promoter Activities in the Presence of HEV Genomic RNA. A recent study showed that HEV infection can induce inflammatory cytokines and chemokines, including CXCL-8 [20]. To determine the minimum region of CXCL-8 promoter required for HEV enhanced CXCL-8 transcription, HuH-7 cells were cotransfected with HEV genomic RNA and CXCL-8 promoterluciferase reporters of different lengths. The HEV genomic RNA can replicate and generate infectious HEV in cell culture [21]. Luciferase activity, as an indicator of CXCL-8 promoter activity, was measured 48 hours after transfection. As shown in Figure 1, we observed substantial luciferase activities when transcribed from the $-1400,-500,-230$, -193 , and -173 CXCL- 8 promoters. The -173 bp promoter displayed the highest promoter activity, suggesting that the -173 promoter contains the sequences necessary for CXCL-8 promoter activation by HEV.

3.2. CXCL-8 Promoter Activation by HEV ORF-1 via AP-1. HEV encodes three proteins. To determine which HEV protein is responsible for activating the CXCL-8 promoter, we 


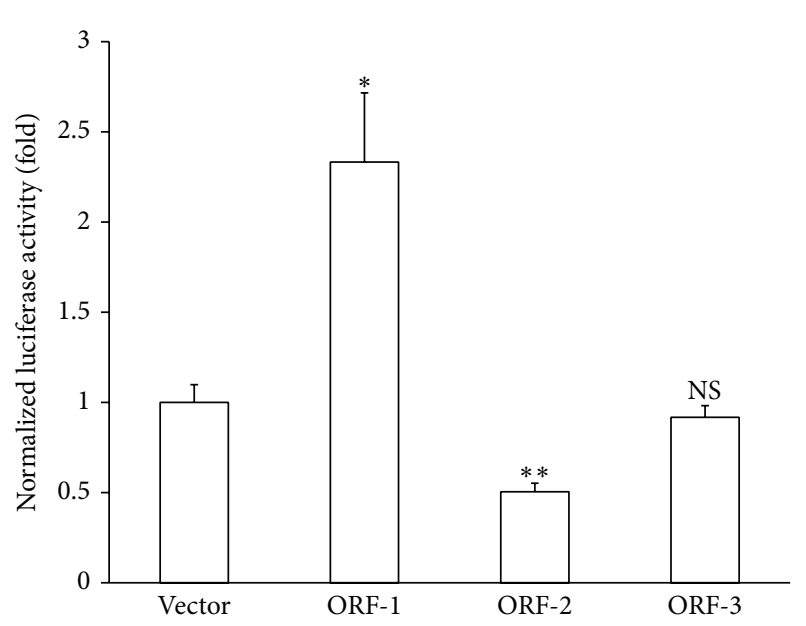

(a)

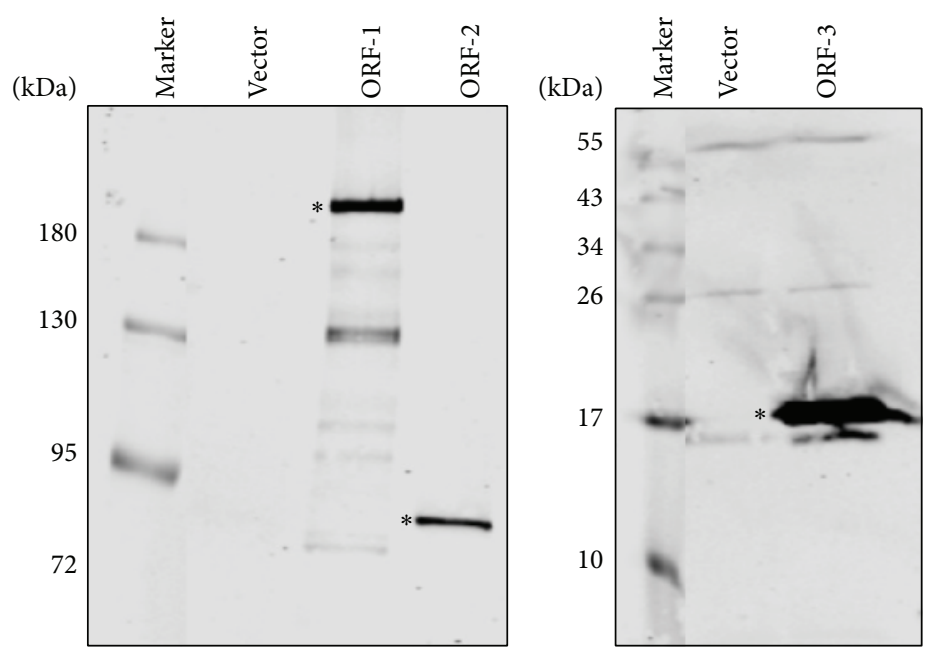

(b)

FIGURE 2: CXCL-8 transcriptional activation by HEV ORF-1. (a) HuH-7 cells were cotransfected with plasmid vector, or plasmids expressing HEV ORF-1, ORF-2, or ORF-3 proteins and the -173 CXCL-8 promoter-luciferase reporter. Luciferase assay was performed 48 hours after transfection. Luciferase activity was normalized against the protein concentration of the same samples. $*: p \leq 0.05, * *: p \leq 0.01$, and NS: not significant by Student's $t$-test. (b) The expression of HEV ORF-1, ORF-2, or ORF-3 proteins with a N-terminal Flag-tag after transfection into HEK293 cells was analyzed by immunoprecipitation followed by Western blotting with an anti-Flag antibody. The location of HEV proteins was indicated by $*$ in the blot.

cotransfected HuH-7 cells with plasmids expressing the ORF1, ORF-2, or ORF-3 proteins, or the vector control, together with the -173 CXCL- 8 promoter reporter. As shown in Figure 2(a), the expression of the ORF-1 protein significantly increased CXCL- 8 promoter activity in comparison to vector control, whereas the ORF-2 protein inhibited and the ORF3 protein had no effect on CXCL- 8 promoter activity. The expression of HEV proteins was confirmed by immunoprecipitation followed by Western blotting (Figure 2(b)). These results demonstrate that HEV ORF-1 protein can activate the CXCL-8 promoter.

The -173 CXCL- 8 promoter contains the binding sequence for AP-1 [23]. To investigate the role of AP-1, the AP-1 binding sequence was removed from the CXCL-8 promoter by mutagenesis. As shown in Figure 3(a), the ORF-1 protein could no longer activate the MAP-1 CXCL-8 promoter. As a control, we used another mutant CXCL-8 promoter with the NRE sequence removed. HEV ORF-1 protein could activate the MNRE CXCL- 8 promoter to a comparable level as to the wild-type CXCL- 8 promoter (Figures 3(a) and 2(a)). These results demonstrate that AP-1 plays a role in CXCL- 8 promoter activation by HEV ORF-1 protein.

3.3. AP-1 Upregulation by HEV ORF-1. Since the AP-1 binding sequence within the CXCL-8 promoter was shown to be required for activation by HEV ORF-1 protein, we hypothesized that HEV ORF-1 protein can directly increase transcription mediated by AP-1. We therefore cotransfected $\mathrm{HuH}-$ 7 cells with a HEV ORF-1 protein expressing plasmid and a luciferase reporter plasmid with seven tandem repeats of the AP-1 binding sequence. Figure 3(b) shows that the expression of HEV ORF-1 protein resulted in greater than threefold increase in the luciferase activity expressed downstream from the tandem AP-1 motifs when compared with basal promoter control. These results suggest that HEV ORF-1 protein can indeed upregulate AP-1-mediated transcription.

3.4. CXCL-8 Promoter Activation by HEV Genomic RNA Requires AP-1. Although we showed that AP-1 is involved in CXCL-8 promoter activation by HEV ORF-1, it was important to demonstrate that transcriptional activation of the CXCL- 8 promoter by HEV also requires AP-1. To address this question, we cotransfected HuH-7 cells with the HEV genomic RNA, together with wild-type or mAP-1 -173 CXCL-8 promoter-luciferase reporters. Results showed that elimination of the AP-1 binding sequence resulted in a significantly lower luciferase activity in comparison to the wild-type promoter (Figure 4). This finding demonstrates the importance of AP-1 in CXCL-8 promoter activation by HEV.

\section{Discussion}

Inflammatory cytokines and chemokines play a critical role in the pathology associated with viral infections. Although elevated inflammatory cytokine/chemokine levels are detected in HEV-associated liver failure patients [26], there is very limited information on how HEV regulates these genes at the molecular level. Devhare et al. showed upregulation of several inflammatory cytokine/chemokine genes and downregulation of interferons after $\mathrm{HEV}$ infection in a cell culture model [20]. Consistently, we showed that transfection of a $\mathrm{HEV}$ genomic RNA is associated with substantial CXCL8 transcription in a promoter-luciferase reporter assay. We further demonstrated that the ORF-1 protein, but not the 


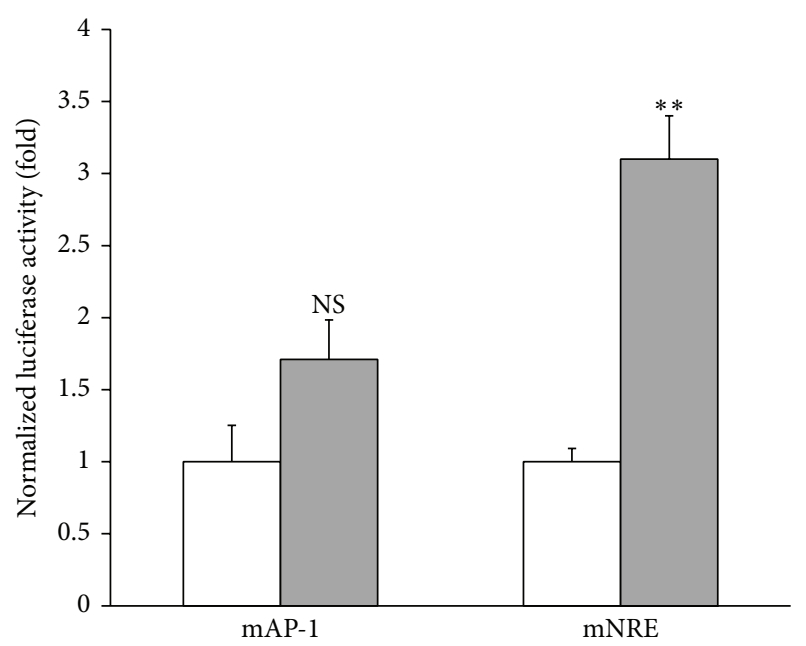

$\square$ Vector

$\square$ ORF-1

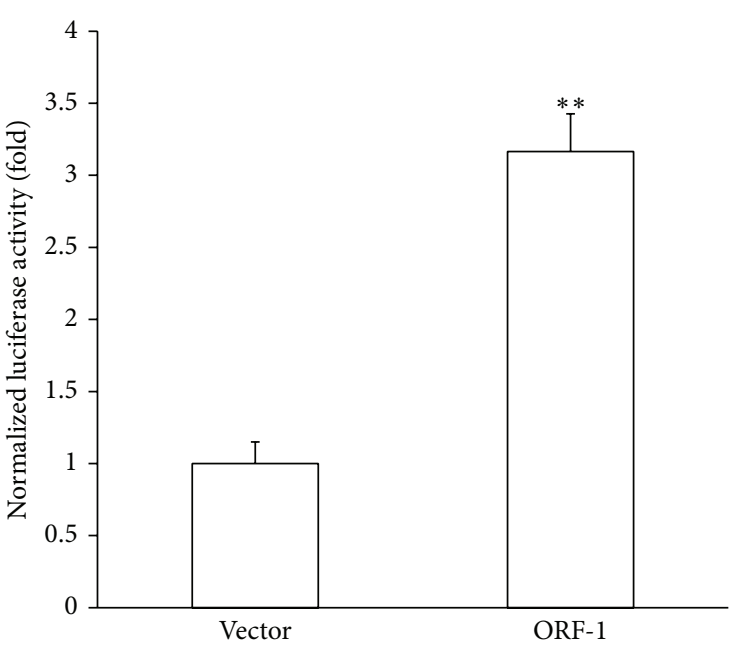

(b)

FIGURE 3: AP-1 is involved in CXCL-8 activation by HEV ORF-1. HuH-7 cells were cotransfected with plasmid vector or a plasmid expressing HEV ORF-1 protein, together with -173 mAP-1 or mNRE CXCL-8 promoter-luciferase reporters (a) or a luciferase reporter with seven repeats of the AP-1 binding sequences (b). Luciferase assay was performed 48 hours after transfection. Luciferase activity was normalized against the protein concentration of the same samples. $* *: p \leq 0.01$; NS: not significant by Student's $t$-test.

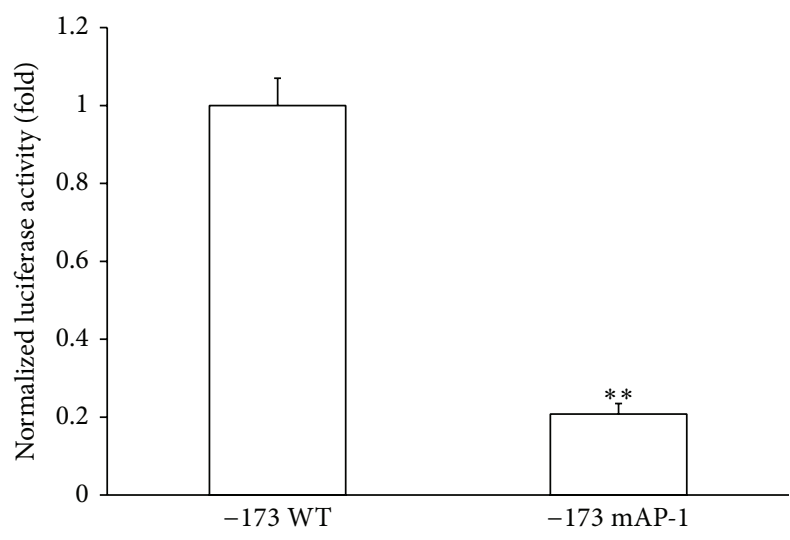

FIGURE 4: AP-1 is required for CXCL-8 activation by HEV genomic RNA. HuH-7 cells were cotransfected with HEV genomic RNA, together with the wild-type or mAP-1 -173 CXCL-8 promoterluciferase reporters. Luciferase assay was performed 48 hours after transfection. Luciferase activity was normalized against the protein concentration of the same samples. $* *: p \leq 0.01$ by Student's $t$-test.

ORF-2 or ORF-3 proteins, can activate CXCL-8 promoter. In addition, we identified the requirement of AP-1 in CXCL-8 transcription activation by both $\mathrm{HEV}$ genomic RNA and the ORF-1 protein.

Amongst the CXCL-8 promoters of different lengths ranging from -1400 to -173 , the -193 and -173 promoters exhibit higher degree of activities in the presence of $\mathrm{HEV}$ RNA (Figure 1). The mechanisms of this differential activation are not fully understood. The activity of a promoter is determined by the interplay of positive and negative factors involved. The binding motifs for multiple transcriptional factors, such as IRF-1, HNF-1, and GR, are located between the -1400 and -173 promoter region [22]. Therefore, it is possible that multiple transcription factors are involved in regulating CXCL-8 transcription by HEV. Furthermore, a few mechanisms, such as histone deacetylation and OCT-1 binding, have been shown to inhibit the CXCL- 8 promoter activity [27]. Whether these mechanisms play a role in CXCL8 promoter regulation by $\mathrm{HEV}$ requires further investigation.

The ORF-1 protein, essential for HEV RNA replication, consists of a few domains which can function as methyltransferase, cysteine protease, RNA helicase, and RNA-dependent RNA polymerase [6]. It would be interesting to determine which domains of ORF-1 are responsible for activating CXCL- 8 transcription. The cysteine protease domain and the adjacent $\mathrm{X}$ domain have been shown to inhibit interferon transcription [28]. Thus, the ORF-1 protein can both upregulate inflammatory chemokine expression and downregulate interferon expression after HEV infection.

The capsid ORF-2 protein, when expressed as an intracellular protein, was found to inhibit CXCL-8 transcription (Figure 2(a)). Previous studies have shown that the UVinactivated HEV virus, but not soluble ORF-2 protein, induces inflammatory cytokines and chemokines [20]. This indicates that the ORF-2 protein has different functional properties as a component of the viral capsid compared with an intracellular protein. Further studies are required to study the functions of the ORF-2 protein.

\section{Conclusions}

We showed that HEV genomic RNA and the ORF-1 protein significantly enhance CXCL-8 promoter activity which 
requires the transcription factor AP-1. This newly identified function of the ORF-1 protein should help understand the molecular mechanisms of HEV-host interactions and HEVassociated pathogenesis.

\section{Disclosure}

This paper is published with the permission of the Director of VIDO-InterVac, Journal Series no. 747.

\section{Conflict of Interests}

The authors declare that there is no conflict of interests regarding the publication of this paper.

\section{Acknowledgments}

The authors would like to thank Dr. Emerson and Dr. Buendia for providing plasmids. The authors would like to thank Dr. Robert Brownlie for proofreading the paper. This work was supported by operating grants from the Canadian Institutes of Health Research and Saskatchewan Health Research Foundation to Qiang Liu. Zhubing Li is a recipient of University of Saskatchewan Vaccinology and Immunotherapeutics Graduate Student scholarship. Lu Chen is supported by the China Scholarship Council.

\section{References}

[1] A. Ahmed, I. A. Ali, H. Ghazal, J. Fazili, and S. Nusrat, "Mystery of hepatitis e virus: recent advances in its diagnosis and management," International Journal of Hepatology, vol. 2015, Article ID 872431, 6 pages, 2015.

[2] I. K. Mushahwar, "Hepatitis E virus: molecular virology, clinical features, diagnosis, transmission, epidemiology, and prevention," Journal of Medical Virology, vol. 80, no. 4, pp. 646-658, 2008.

[3] J. Zhang, S. W. Li, T. Wu, Q. Zhao, M. H. Ng, and N. S. Xia, "Hepatitis E virus: neutralizing sites, diagnosis, and protective immunity," Reviews in Medical Virology, vol. 22, pp. 339-349, 2012.

[4] M. T. Pérez-Gracia, M. L. M. Lindemann, and M. C. M. Villalba, "Hepatitis E: current status," Reviews in Medical Virology, vol. 23, no. 6, pp. 384-398, 2013.

[5] R. Aggarwal and S. Naik, "Epidemiology of hepatitis E: current status," Journal of Gastroenterology and Hepatology, vol. 24, pp. 1484-1493, 2009.

[6] N. Kamar, H. R. Dalton, F. Abravanel, and J. Izopet, "Hepatitis E virus infection," Clinical Microbiology Reviews, vol. 27, no. 1, pp. 116-138, 2014.

[7] N. Pavio, X.-J. Meng, and V. Doceul, "Zoonotic origin of hepatitis E," Current Opinion in Virology, vol. 10, pp. 31-41, 2015.

[8] R. Aggarwal and S. Jameel, "Hepatitis E," Hepatology, vol. 54, no. 6, pp. 2218-2226, 2011.

[9] N. Kamar, R. Bendall, F. Legrand-Abravanel et al., "Hepatitis E," The Lancet, vol. 379, no. 9835, pp. 2477-2488, 2012.

[10] M. T. Perez-Gracia, B. Suay, and M. L. Mateos-Lindemann, "Hepatitis E: an emerging disease," Infection, Genetics and Evolution, vol. 22, pp. 40-59, 2014.
[11] S. Kumar, S. Subhadra, B. Singh, and B. K. Panda, "Hepatitis E virus: the current scenario," International Journal of Infectious Diseases, vol. 17, pp. e228-e233, 2013.

[12] F. Abravanel, S. Lhomme, M. Dubois et al., "Hepatitis E virus," Medecine et Maladies Infectieuses, vol. 43, no. 7, pp. 263-270, 2013.

[13] H. Ghasemi, T. Ghazanfari, R. Yaraee, S. Faghihzadeh, and Z. M. Hassan, "Roles of IL-8 in ocular inflammations: a review," Ocular Immunology and Inflammation, vol. 19, no. 6, pp. 401412, 2011.

[14] M. Rotondi, F. Coperchini, and L. Chiovato, "CXCL8 in thyroid disease: from basic notions to potential applications in clinical practice," Cytokine and Growth Factor Reviews, vol. 24, no. 6, pp. 539-546, 2013.

[15] F. Lotti and M. Maggi, "Interleukin 8 and the male genital tract," Journal of Reproductive Immunology, vol. 100, no. 1, pp. 54-65, 2013.

[16] A. Pichert, D. Schlorke, S. Franz, and J. Arnhold, "Functional aspects of the interaction between interleukin- 8 and sulfated glycosaminoglycans," Biomatter, vol. 2, no. 3, pp. 142-148, 2012.

[17] P. Zarogoulidis, F. Katsikogianni, T. Tsiouda, A. Sakkas, N. Katsikogiannis, and K. Zarogoulidis, "Interleukin-8 and interleukin-17 for cancer," Cancer Investigation, vol. 32, no. 5, pp. 197-205, 2014.

[18] N. Todorović-Raković and J. Milovanović, "Interleukin-8 in breast cancer progression," Journal of Interferon \& Cytokine Research, vol. 33, no. 10, pp. 563-570, 2013.

[19] D. Gales, C. Clark, U. Manne, and T. Samuel, "The chemokine CXCL8 in carcinogenesis and drug response," ISRN Oncology, vol. 2013, Article ID 859154, 8 pages, 2013.

[20] P. B. Devhare, S. N. Chatterjee, V. A. Arankalle, and K. S. Lole, "Analysis of antiviral response in human epithelial cells infected with hepatitis E virus," PLoS ONE, vol. 8, no. 5, Article ID e63793, 2013.

[21] P. Shukla, H. T. Nguyen, K. Faulk et al., "Adaptation of a genotype 3 hepatitis $\mathrm{E}$ virus to efficient growth in cell culture depends on an inserted human gene segment acquired by recombination," Journal of Virology, vol. 86, no. 10, pp. 56975707, 2012.

[22] L. Lévy, C. Neuveut, C.-A. Renard et al., “Transcriptional activation of interleukin- 8 by $\beta$-catenin-Tcf4," Journal of Biological Chemistry, vol. 277, no. 44, pp. 42386-42393, 2002.

[23] K. Yasumoto, S.-I. Okamoto, N. Mukaida, S. Murakami, M. Mai, and K. Matsushima, "Tumor necrosis factor $\alpha$ and interferon $\gamma$ synergistically induce interleukin 8 production in a human gastric cancer cell line through acting concurrently on AP-1 and NF-kB-like binding sites of the interleukin 8 gene," The Journal of Biological Chemistry, vol. 267, no. 31, pp. 22506-22511, 1992.

[24] M. Nourbakhsh, S. Kälble, A. Dörrie, H. Hauser, K. Resch, and M. Kracht, "The NF- $\kappa$ B repressing factor is involved in basal repression and interleukin (IL)-1-induced activation of IL- 8 transcription by binding to a conserved NF- $\kappa$ B-flanking sequence element," The Journal of Biological Chemistry, vol. 276, no. 6, pp. 4501-4508, 2001.

[25] F. L. Graham and A. J. van der Eb, "A new technique for the assay of infectivity of human adenovirus 5 DNA," Virology, vol. 52, no. 2, pp. 456-467, 1973.

[26] L. J. Krain, K. E. Nelson, and A. B. Labrique, "Host immune status and response to hepatitis E virus infection," Clinical Microbiology Reviews, vol. 27, pp. 139-165, 2014. 
[27] E. Hoffmann, O. Dittrich-Breiholz, H. Holtmann, and M. Kracht, "Multiple control of interleukin-8 gene expression," Journal of Leukocyte Biology, vol. 72, no. 5, pp. 847-855, 2002.

[28] Y. Nan, Y. Yu, Z. Ma, S. K. Khattar, B. Fredericksen, and Y. J. Zhang, "Hepatitis E virus inhibits type I interferon induction by ORF1 products," Journal of Virology, vol. 88, pp. 11924-11932, 2014. 


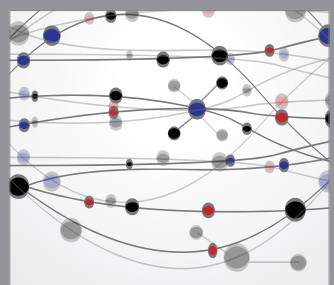

The Scientific World Journal
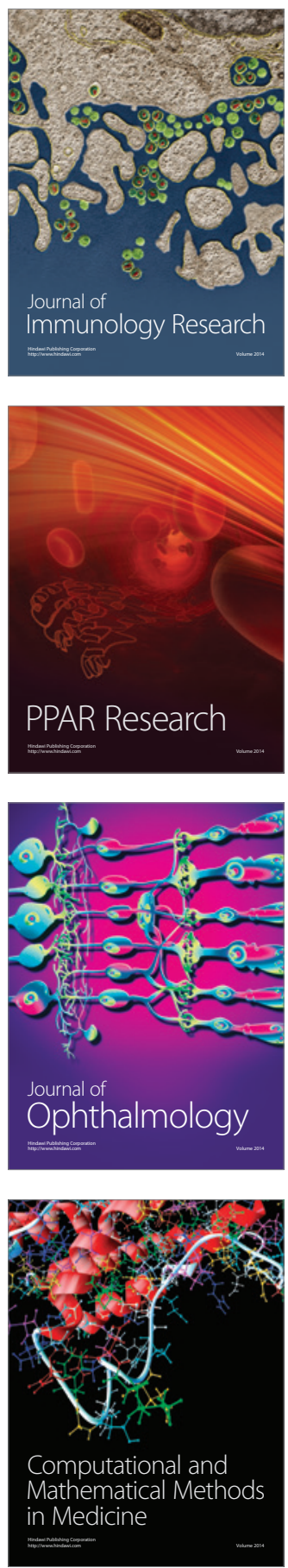

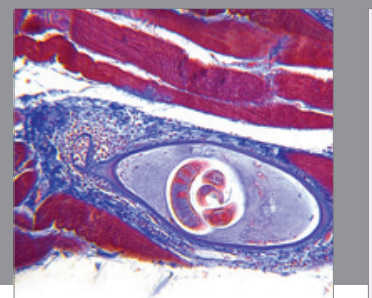

Gastroenterology

Research and Practice
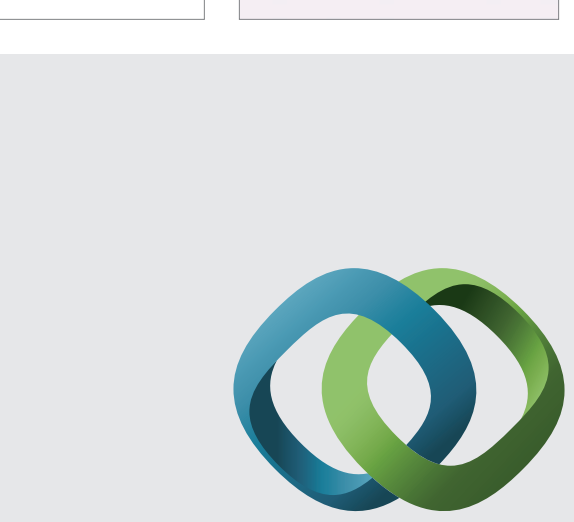

\section{Hindawi}

Submit your manuscripts at

http://www.hindawi.com
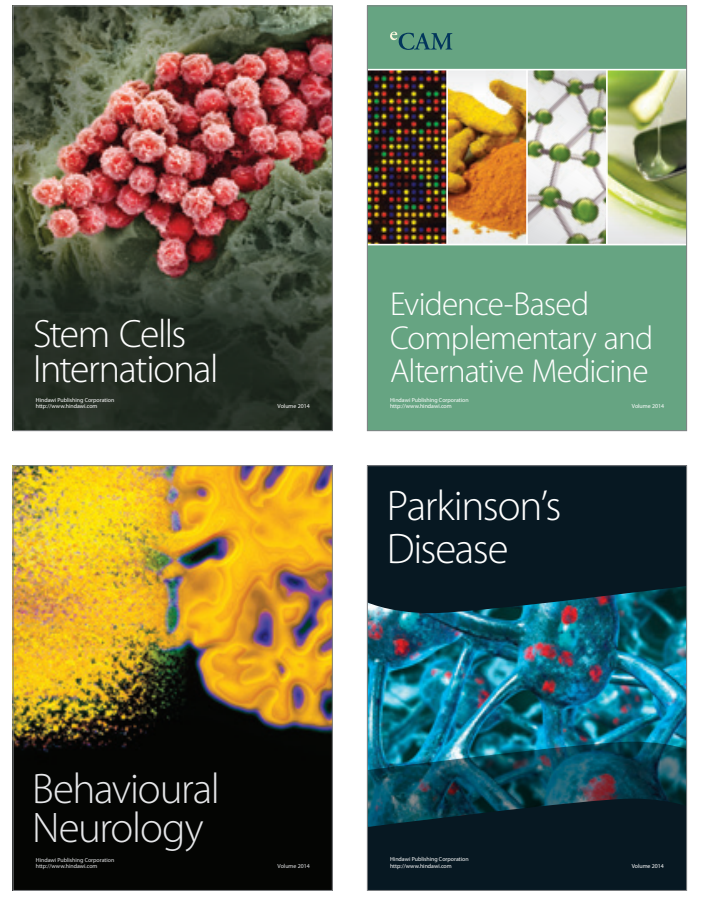
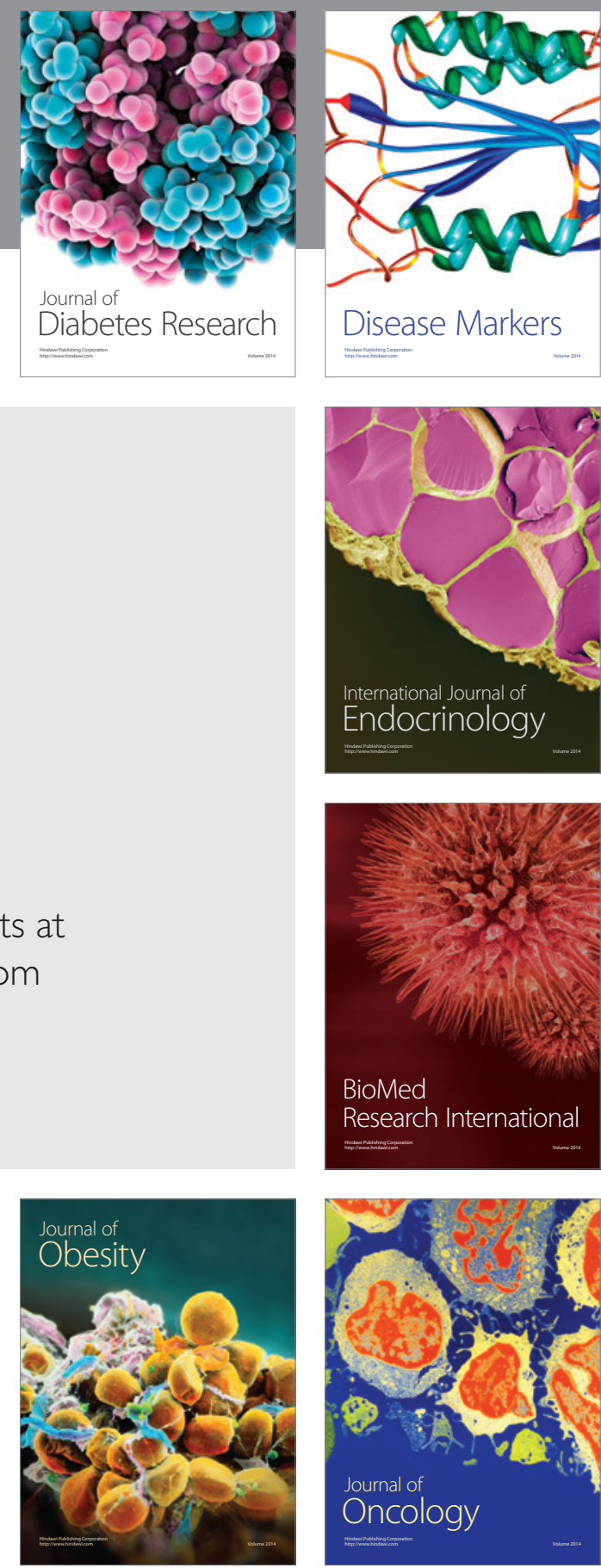

Disease Markers
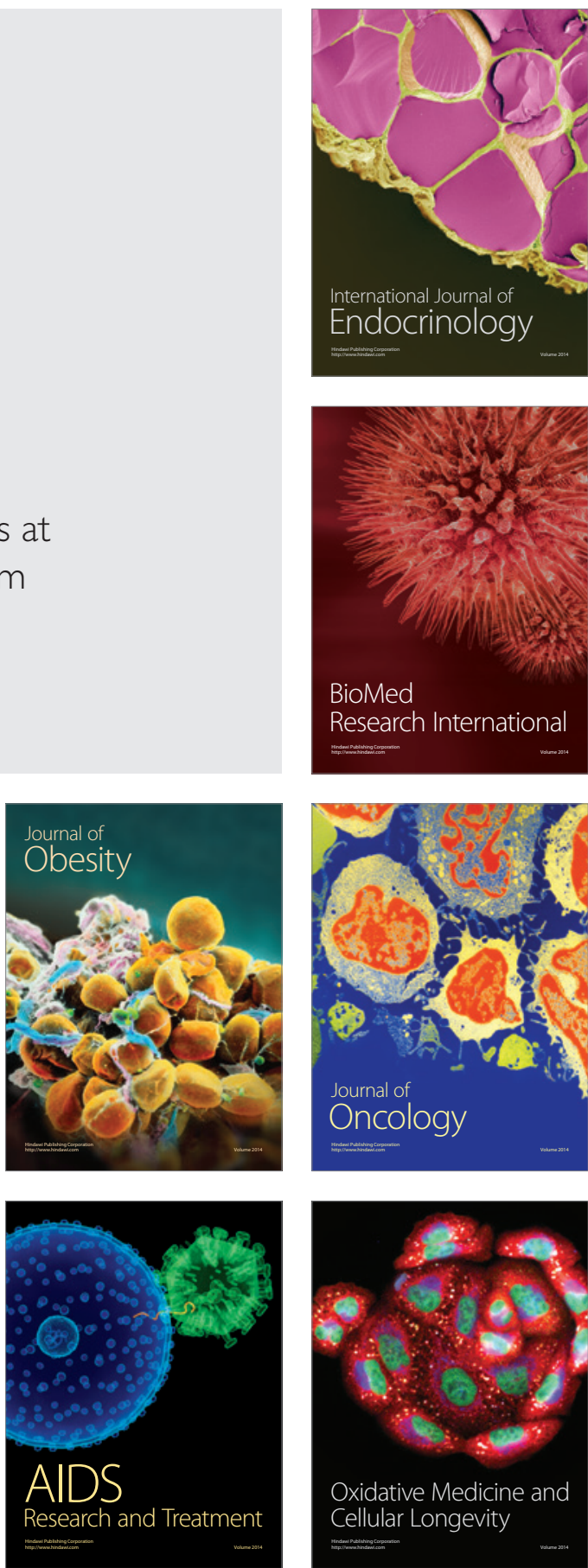